\title{
ON STRICTLY CYCLIC ALGEBRAS, RALGEBRAS AND REFLEXIVE OPERATORS
}

\author{
BY \\ DOMINGO A. HERRERO(1) AND ALAN LAMBERT
}

\begin{abstract}
An operator algebra $\mathfrak{U} \subset \mathcal{L}(X)$ (the algebra of all operators in a Banach space $X$ over the complex field $C$ ) is called a "strictly cyclic algebra" (s.c.a.) if there exists a vector $x_{0} \in X$ such that $\mathfrak{A}\left(x_{0}\right)=\left\{A x_{0}: A \in \mathfrak{A}\right\}=X ; x_{0}$ is called a "strictly cyclic vector" for $\mathfrak{A}$. If, moreover, $x_{0}$ separates elements of $\mathfrak{X}$ (i.e., if $A \in \mathfrak{U}$ and $A x_{0}=0$, then $A=0$ ), then $\mathfrak{A}$ is called a "separated s.c.a."

$\mathscr{X}$ is a $\mathfrak{P}_{\text {algebra }}$ if, given $x_{1}, \ldots, x_{n} \in \mathcal{X}$, there exists $x_{0} \in X$ such that $\left\|A x_{j}\right\|$ $\leq\left\|A x_{0}\right\|$, for all $A \in \mathfrak{A}$ and for $j=1, \ldots, n$. Among other results, it is shown that if the commutant $\mathfrak{X}^{\prime}$ of the algebra $\mathfrak{X}$ is an s.c.a., then $\mathfrak{A}$ is a $\mathfrak{P}$-algebra and the strong and the uniform operator topology coincide on $\mathfrak{A}$; these results are specialized for the case when $\mathfrak{U}$ and $\mathfrak{X}^{\prime}$ are separated s.c.a.'s. (Here, and in what follows, algebra means strongly clased subalgebra on $\mathcal{L}(X)$ containing the identity $I$ on $X$.)

In the second part of the paper, it is shown that a large class of bilateral weighted shifts (which includes all the invertible ones) in a Hilbert space are reflexive. The result is used to show that "reflexivity" is neither a "restriction property" nor a "quotient property."

Recall that an algebra $\mathfrak{X}$ is called reflexive if, whenever $T \in \mathcal{L}(X)$ and the lattice of invariant subspaces of $T$ contains the corresponding lattice of $\mathfrak{x}$, then $T \in \mathfrak{x}$. (2)
\end{abstract}

1. A strictly cyclic algebra and its commutant. Let $\mathfrak{A}$ be a strictly cyclic algebra on $X$, with strictly cyclic vector $x_{0}$. We have a commutative diagram:

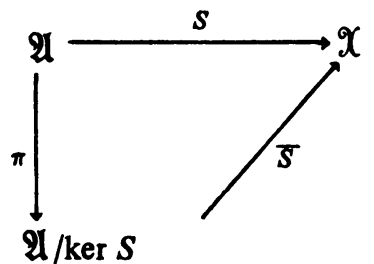

Received by the editors September 17, 1971 and, in revised form, June 10, 1972.

AMS (MOS) subject classifications (1970). Primary 46L20, 47A15; Secondary. 47A25, 47B20.

Key words and phrases. Banach algebras, algebras of operators, P-algebras, strictly cyclic algebras, reflexive algebra, weighted shifts, invariant subspaces.

(1) Research supported by National Science Foundation grant GU 3171.

(2) After this paper was written, the authors received the preprint Notes on strictly cyclic operator algebras by Mary R. Embry. In that paper, M.R. Embry found independent proofs of Theorem 1(i) and Theorem 3 below. 
where $S(A)=A x_{0}$, ker $S$ is a closed left ideal in $\mathfrak{A}, \pi$ is the canonical projection onto the quotient space and $\bar{S}(S=\bar{S} \bullet \pi)$ is the quotient map. Clearly, $S$ is continuous, linear and onto, $\pi$ has the same properties and $\bar{S}$ is an isomorphism of Banach spaces. Moreover, $\pi$ (and therefore $S$ ) is an open map.

Theorem 1. Let $\mathfrak{A}$ be a subalgebra of $\mathcal{L}(\mathcal{X})$ and let $\mathfrak{U}^{\prime}=\{T \in \mathcal{L}(\mathcal{X})$ : TA $=A T$, for all $A \in \mathfrak{A}\}$ be the commutant of $\mathfrak{A}$. Assume that $\mathfrak{A}^{\prime}$ has an s.c. vector $x_{0}$; then

(i) $\mathfrak{A}$ has the property $(\mathcal{P})$ (i.e., $\mathfrak{A}$ is a $\mathscr{P}$-algebra);

(ii) the strong and the uniform operator topologies coincide on $\mathfrak{A}$.

Proof. (i) By hypothesis, $X=\mathfrak{X}^{\prime} x_{0}$. Thus, given $x_{0}, \ldots, x_{n} \in X$, there exist $A_{1}, \ldots, A_{n} \in \mathfrak{A}^{\prime}$ such that $x_{j}=A_{j} x_{0}$ for $j=1, \ldots, n$. Moreover, since $S$ is an open map, the $A_{j}$ 's can be chosen in such a way that $\left\|A_{j}\right\| \leq C\left\|x_{j}\right\|$, where $C$ is a positive constant, independent of $j$ (see [5, Lemma 1]).

Thus, if $B \in \mathfrak{A}$, then

$$
\left\|B x_{j}\right\|=\left\|B A_{j} x_{0}\right\|=\left\|A_{j} B x_{0}\right\| \leq\left\|A_{j}\right\| \cdot\left\|B x_{0}\right\| \leq\left\|B\left(M x_{0}\right)\right\|,
$$

where $M=C \max \left\{\left\|x_{j}\right\|: j=1, \ldots, n\right\}$.

Hence, $\mathfrak{A}$ is a $\mathcal{P}$-algebra.

(ii) Let $\left\{A_{\lambda}: \lambda \in \Lambda\right.$, a directed set $\}$ be a net in $\mathscr{A}$ converging strongly to $A$ $(\in \mathfrak{A})$. Let $x=B x_{0}, B \in \mathfrak{A}^{\prime}$ be chosen s.t. $\|x\| \leq 1$ and $\|B\| \leq C$. Then

$$
\left\|\left(A_{\lambda}-A\right) x\right\|=\left\|\left(A_{\lambda}-A\right) B x_{0}\right\|=\left\|B\left(A_{\lambda}-A\right) x_{0}\right\| \leq C\left\|\left(A_{\lambda}-A\right) x_{0}\right\| .
$$

Therefore

$$
\lim _{\lambda \in \Lambda}\left\|A_{\lambda}-A\right\| \leq C \lim _{\lambda \in \Lambda}\left\|\left(A_{\lambda}-A\right) x_{0}\right\|=0 ;
$$

i.e., strongly convergent nets are actually norm-convergent. It follows immediately that the strong and the uniform topologies coincide on $\mathfrak{A}$.

Now, most of the results of $[6,83]$ can be "translated" to these algebras (or sums of them). Consider, in particular, the four algebras associated with an operator $T \in \mathcal{L}(\mathcal{X})$ defined in [6]; i.e., the algebras $\mathfrak{A}_{T}$ and $\mathfrak{U}_{T}^{a}$ generated by the polynomials and by the rational functions (with poles outside of the spectrum) in $T$, resp., and the commutant $\mathfrak{X}_{T}^{\prime}$ and the double commutant $\mathfrak{A}_{T}^{\prime \prime}$ of $T$. Then $\mathfrak{A}_{T} \subset \mathfrak{U}_{T}^{a} \subset \mathfrak{U}_{T}^{\prime \prime} \subset \mathfrak{U}_{T}^{\prime}$, and the corresponding lattices of invariant subspaces satisfy the reverse inclusions. The above mentioned results yield the following

Corollary 1. If $T \in \mathcal{L}(\mathcal{X})$ and $\mathfrak{A}_{T}^{\prime}$ is an s.c.a., then any two of the algebras $\mathfrak{A}_{T}$, $\mathfrak{U}_{T}^{a}, \mathfrak{X}_{T}^{\prime \prime}$ and $\mathfrak{X}_{T}^{\prime}$ are equal if and only if the corresponding lattices of invariant subspaces are equal.

Let $\left\{x_{1}, x_{2}\right\}$ be linearly independent in $\chi$; if $x_{0}$ is any nonzero vector in $\chi$, then (by the Hahn-Banach theorem) there exists a continuous linear functional $x^{*}$ on 
$\chi$ such that $x^{*}\left(x_{0}\right)=1$. If $\propto M=\operatorname{ker}\left(x^{*}\right)$, then $\chi=\propto M \oplus\left\{\lambda x_{0}: \lambda \in \mathbf{C}\right\}$ and the linear map $T_{0}$ defined by $\left.T_{0}\right|_{\mathcal{M}}=\left.I\right|_{\mathcal{L}}, T_{0} x_{0}=0$, belongs to $\mathcal{L}(\mathcal{X})$. Moreover, it is clear that either $T_{0} x_{1} \neq 0$ or $T_{0} x_{2} \neq 0$; thus we have the following

Lemma 2. If $\operatorname{dim} \chi>1$, then $\mathcal{L}(\mathcal{X})$ does not have the property $(\mathfrak{P})$.

Hence, $\mathfrak{A}=\{\lambda I: \lambda \in \mathbf{C}\}$ is a $\mathcal{P}$-algebra whose commutant $\mathfrak{U}^{\prime}=\mathcal{L}(\mathcal{X})$ is an s.c.a., but not a $\mathscr{P}$-algebra $(\operatorname{dim} \mathcal{X}>1)$. Here is an open problem: Assume that $\mathfrak{U}$ is a $\mathfrak{P}$-algebra. Does it imply that $\mathfrak{U}^{\prime \prime}$ is a $\mathfrak{P}$-algebra too?

The results of [5] can be "specialized" here. In fact, we have

Theorem 2. Let $\mathfrak{A}$ be an s.c.a. with strictly cyclic vector $x_{0}$. Then every cyclic vector of $\mathfrak{A}$ is actually a strictly cyclic vector. Moreover, the set of all these vectors is open in $\mathcal{X}$ and coincides with $C_{\mathfrak{\Re}}=\left\{A x_{0}: A \in \mathfrak{A}\right.$ and $A$ has a left inverse $(\bmod \operatorname{ker} S)$ in $\mathfrak{A}\}$, where $\operatorname{ker} S$ is the kernel of the map $S$ defined above.

Proof. If $x$ is a cyclic vector of $\mathfrak{A}$, then $\mathfrak{A} x$ is a dense linear manifold of $\mathcal{X}$ invariant under $\mathfrak{A}$. Since, according to [5, Lemma 2] the only linear manifold of $\chi$ satisfying these two conditions is $\chi$ itself, we conclude that $\mathfrak{A} x=\chi$. In other words, $x$ is a strictly cyclic vector.

Clearly, if $x=A x_{0} \in C_{\mathfrak{U}}$, then there exists $B \in \mathfrak{A}$ such that $B A=I+L$, for some $L \in \operatorname{ker} S$. Therefore, $x_{0}=B A x_{0} \in \mathfrak{A} x$; hence, $\mathcal{X}=\mathfrak{A} x_{0} \subset \mathfrak{A} x$, which implies that $x$ is a strictly cyclic vector of $\mathfrak{A}$.

Conversely, if $x=A x_{0}$ is cyclic (and therefore, it is strictly cyclic), then

$$
I x_{0}=x_{0} \in \mathcal{X}=\mathfrak{A} x=\left\{B A x_{0}: B \in \mathfrak{A}\right\} \text {. }
$$

Therefore, there exists $B \in \mathfrak{A}$ s.t. $(I-B A) x_{0}=0$; i.e., $I-B A=L$ $\in$ ker $S$. Hence $B A=I-L$, i.e., $B$ is a left inverse of $A$, modulo ker $S$. Therefore $\chi \in C_{\mathfrak{r}}$.

Finally, to see that $C_{\mathfrak{Y}}$ is an open subset of $\chi$, observe that the mapping $S$ is open and (see, for example, [10, Chapter I]) $S^{-1}\left(C_{\mathfrak{U}}\right)$ is open in $\mathfrak{A}$.

\section{Separated strictly cyclic algebras.}

Lemma 3 [10, p. 3]. Let $\mathfrak{B}$ be a Banach algebra with identity e; let $\mathfrak{B}_{L}=\left\{L_{b}=\right.$ left multiplication by $b ; b \in \mathfrak{B}\}$ and let $\mathfrak{B}_{R}$ (right multiplications) be similarly defined (i.e., $b \rightarrow L_{b}$ and $b \rightarrow R_{b}$ are the regular left and right, resp., representations of $\mathfrak{B})$. Then:

(i) $\mathfrak{B}_{L}$ and $\mathfrak{B}_{R}$ are separated s.c.a.'s of $\mathcal{L}(\mathfrak{B})$ (i.e., $I \in \mathfrak{B}_{L}, I \in \mathfrak{B}_{A}$ and $\mathfrak{B}_{L}, \mathfrak{B}_{R}$ are strongly closed in $\mathcal{L}(\mathfrak{B})$ ).

(ii) $\mathfrak{B}_{L}^{\prime}=\mathfrak{B}_{R} ; \mathfrak{B}_{R}^{\prime}=\mathfrak{B}_{L}$.

Theorem 3. Let $\mathfrak{A}$ be a separated s.c.a.; then

(i) $\mathfrak{U}^{\prime}$ is also a separated s.c.a.;

(ii) $\mathfrak{U}^{\prime \prime}=\mathfrak{U}$;

(iii) $\mathfrak{A}$ and $\mathfrak{X}^{\prime}$ have the property $(\mathfrak{P})$. 
Proof. Let $\mathfrak{A}$ be a separated s.c.a. with separating strictly cyclic vector $x_{0}$; then (see [8, Lemma 2.2]) there exists a constant $C \geq 1$ s.t., if $A_{x} \in \mathfrak{A}$ is the (unique!) operator satisfying $A_{x} x_{0}=x$, then $\|x\| \leq\left\|A_{x}\right\| \leq C\|x\|$.

These two inequalities show that $\mathfrak{U}$ is isomorphic (as a Banach space) with $\chi$. Moreover, if for $x, y \in X$ the product $x \cdot y$ is defined by

$$
x \cdot y=A_{x} \cdot A_{y} x_{0}
$$

and $\mathcal{X}$ is re-normed with $\|x\|^{\prime}=\left\|A_{x}\right\|$, then $\mathfrak{B}=\left(\mathcal{X},+, \cdot\|\cdot\|^{\prime}\right)$ is a Banach algebra with identity $x_{0},\|\cdot\|^{\prime}$ is equivalent to $\|\cdot\|,\left\|x_{0}\right\|^{\prime}=1$ and $\mathfrak{A}$ can be identified with $\mathfrak{B}_{L}$. In other words: a separated s.c.a. is essentially the algebra of all "left multiplications" in a Banach algebra with identity. Using this characterization and Lemma 3, we obtain (i) and (ii).

Finally, using (i), (ii) and Theorem l(i), we obtain (iii).

Thus, if $\mathfrak{A}$ is a separated s.c.a., Theorem 2 applies both to $\mathfrak{A}$ and $\mathfrak{A}^{\prime}$. Moreover, in this case we also have

Corollary 4. Let $\mathfrak{A}$ be a separated s.c.a.; then

(i) $\mathfrak{A}$ and $\mathfrak{U}^{\prime}$ have the same separating cyclic vectors (which are actually strictly cyclic vectors for both algebras); this set of vectors is open in $\chi$ and can be identified with

$$
S C=\left\{A x_{0}: A \in \mathfrak{A} \text { and } A \text { is invertible }\right\}
$$

where $x_{0}$ is an arbitrary separating s.c. vector of $\mathfrak{A}$.

(ii) Let $A_{x} \in \mathfrak{U}, A_{x}^{\prime} \in \mathfrak{U}^{\prime}$ be the operators defined by

$$
A_{x} x_{0}=A_{x}^{\prime} x_{0}=x
$$

then $\sigma\left(A_{x}\right)=\sigma\left(A_{x}^{\prime}\right)(\sigma(T)=$ spectrum of $T)$.

Proof. (i) Identify $\mathcal{X}$ with a Banach algebra $\mathfrak{B}$ with identity $e=x_{0}$ as in Theorem 3. Then $S C$ is the set of all invertible elements of $\mathfrak{B}$ and, clearly, every element of $S C$ is a separating strictly cyclic vector of $\mathfrak{B}_{L}$.

On the other hand, if $f \in \mathfrak{B}$ is a separating s.c. vector of $\mathfrak{B}_{L}$, then Theorem 2 says that $f$ must have a left inverse in $\mathfrak{B}$. If $f$ is actually invertible, then $f \in S C$, and we are done. Otherwise, there exist two different elements, $b, c \in \mathfrak{B}$, s.t. $b f=c f=e$, and therefore

$$
0=\left(L_{b}-L_{c}\right) f=\left(L_{b}-L_{c}\right) L_{f} e=0 \cdot e,
$$

even when $\left(L_{b}-L_{c}\right) \neq 0$. Hence, $f$ does not separate points of $\mathfrak{B}_{L}$, contradicting our assumption.

Similarly, we can prove that the set of all separating s.c. vectors of $\mathfrak{U}^{\prime}$ also coincides with $S C$. 
That $S C$ is open in $\mathfrak{B}$ is immediate (see [10, Chapter I]).

(ii) Using the above identification, consider the following statements:

(1) $\lambda \in \sigma\left(L_{b}\right)$;

(2) $L_{b}-\lambda I$ has no inverse in $\mathfrak{B}_{L}$;

(3) $b-\lambda e$ has no inverse in $\mathfrak{B}$;

(4) $R_{b}-\lambda I$ has no inverse in $\mathfrak{Y}_{R}$;

(5) $\lambda \in \sigma\left(R_{b}\right)$.

It is not hard to check that each of these statements is equivalent to the next one. Therefore, $\sigma\left(L_{b}\right)=\sigma\left(R_{b}\right)$.

Remarks. (a) The definition of "algebra with the property $(\mathcal{P})$ " was introduced in [6]. For "strictly cyclic algebras," see [7], [9], [10].

(b) If $\mathfrak{A}$ is a separated s.c.a., then $\mathfrak{A}^{\prime \prime}=\mathfrak{A}$ (by Theorem 3). Therefore, if $A \in \mathfrak{A}$ is invertible in $\mathcal{L}(\mathcal{X})$, then $A^{-1} \in \mathfrak{A}$. However, if $A$ has only a left (or right) inverse $B \in \mathcal{L}(\mathcal{X})$, but $A$ is not invertible, then $B \notin \mathfrak{A}$, in general.

For example (see [8]), if $\mathcal{X}$ is a Hilbert space with orthonormal basis $\left\{e_{n}\right\}(n \geq 0)$ and $T$ is the unilateral weighted shift defined by $T e_{n}=$ $(n+2) /(n+1) e_{n+1}$, then $\mathfrak{A}=\mathfrak{A}_{T}=\mathfrak{A}_{T}^{\prime}$ is a maximal abelian separated s.c.a. and $T$ has a right inverse $L \in \mathcal{L}(\mathcal{X})$; but it is clear (since $L$ does not commute with $T$ !) that $L \notin \mathfrak{A}$.

3. Reflexive bilateral weighted shifts. Examples of separated s.c.a.'s generated by a unilateral weighted shift, or by an invertible bilateral weighted shift and its inverse, can be found in [2], [8], [9]. In particular, the "Donoghue weighted shift" $D$ in the Hilbert space of $\left(D\right.$ is defined by $\left.D e_{n}=2^{-n} e_{n+1}, n=0,1,2, \ldots\right)$ generates the maximal abelian separated s.c.a. $\mathfrak{A}_{D}$. This example shows that a $\mathfrak{P}$ algebra need not be a reflexive one. (Furthermore, $\left\{2^{n(n-1) / 2} D^{n}\right\} n=1,2, \ldots$, converges weakly to 0 ; but this sequence does not converge in the strong topology of $\mathcal{L}(\mathcal{X})$. Hence, "strong" cannot be replaced by "weak" in Theorem 1.)

We want to show here that this is no longer the case if $D$ is replaced by a bilateral weighted shift $T$ such that $T$ and $T^{-1}$ generate a separated s.c.a. In fact, our result includes a more general class of shift operators.

In [1], J.A. Deddens remarked that, using the same techniques as in D. Sarason's paper [11], it is possible to prove that a unilateral weighted shift on a Hilbert space with nontrivial (i.e., other than 0 ) compression spectrum is reflexive. This result can be extended to a class of bilateral weighted shifts, as follows:

Theorem 4. Let $B$ be a bilateral weighted shift in a Hilbert space of with ONB $\left\{e_{n}\right\}(-\infty<n<+\infty)$, defined by $B e_{n}=a_{n} e_{n+1}$, where $\left\{a_{n}\right\}(-\infty<n<+\infty)$ is a bounded sequence of positive reals. Assume that either

$$
I^{+}=\liminf _{n \rightarrow+\infty}\left(\prod_{j=0}^{n-1} a_{j}\right)^{1 / n}>0
$$


or

$$
Q^{-}=\liminf _{n \rightarrow+\infty}\left(\prod_{j=1}^{n} a_{-j}\right)^{1 / n}>0
$$

Then $B$ is reflexive.

Proof. Let $L$ be a bounded operator in $\mathcal{H}$ and assume that $\mathcal{\partial}_{L} \supset \mathcal{J}_{B}$ (where $\mathcal{J}$ denotes the lattice of invariant subspaces). Assume that $I^{+}>0$ and for each integer $m$, let $\mathcal{A}_{m}$ be the closed linear span of $\left\{e_{n}: n \geq m\right\}$. The restriction $\left.B\right|_{d m}$ of $B$ to $\mathscr{H}_{m}$ is a unilateral weighted shift in $\alpha_{m}$ with nontrivial compression spectrum. In fact, the compression spectrum of $\left.B\right|_{\text {dim }}$ contains the open disc of radius $I^{+}$(see [2], [4]). It follows from [1] and [12] that

$$
\left.L\right|_{d t_{m}}=\text { (strong) } \lim _{N \rightarrow+\infty} \sum_{n=0}^{N}\left(1-\frac{n}{N+1}\right) c_{n}\left(\left.B\right|_{\alpha \alpha_{k}}\right)^{n}
$$

where the "Taylor coefficients" $c_{n}(n \geq 0)$ are uniquely determined by $L$. Moreover, since $L$ maps $\mathcal{H}_{m}$ into itself for each $m$, it also follows that $L$ commutes with $B$ on the dense linear manifold $U\left\{\alpha_{m}:-\infty<m<+\infty\right\}$ of $\alpha$; hence $L B=B L$ and therefore (by a slight modification of the proof given in [3]),

$$
L=\lim _{N \rightarrow+\infty} \sum_{n=-N}^{+N}\left(1-\frac{n}{N+1}\right) c_{n}^{\prime} B^{n} \quad \text { (in the strong topology), }
$$

where the "Laurent coefficients" $\left\{c_{n}^{\prime}\right\}(-\infty<n<+\infty)$ are uniquely determined by $L$. Since $\left.L\right|_{d m}=P_{m} L P_{m}=L P_{m}$, where $P_{m}$ denotes the orthogonal projection of $\&$ onto $\mathcal{H}_{m}$, multiplying (2) by $P_{m}$ on both sides and comparing this expression with (1) it follows that $c_{n}^{\prime}=c_{n}$, for all $n \geq 0, c_{n}^{\prime}=0$, for $n<0$ and $L \in \mathfrak{U}_{B}$, the strong closure of the polynomials in $B$. In other words, $\mathfrak{A}_{B}$ is a reflexive algebra.

If, instead of $I^{+}>0$ we assume that $Q^{-}>0$, the result can be proved in the same way, replacing $B$ by $B^{*}$ and observing (see [1]) that $\mathfrak{A}_{B}$ is reflexive if and only if $\mathfrak{A}_{B^{*}}$ is reflexive.

Observe that, in the proof of Theorem 4 , we do not use the fact that $\mathcal{I}_{L}$ contains all the invariant subspaces of $B$; in fact, we merely need to assume that $\mathcal{\partial}_{L}$ contains every $M \in \mathcal{J}_{B}$ such that $M \subset \mathcal{H}_{m}$ for some integer $m$. If $B$ is an invertible bilateral weighted shift, then $I^{+}(B)>0$ and $Q^{-}(B)>0$ (see [2], [4]) and 0 belongs to the spectrum of $\left.B\right|_{\mathcal{M}}$ for any invariant subspace $M$ which is contained in $\mathcal{A}_{m}$, for some $m$. Since $B$ is invertible, $M \notin \mathcal{J}_{B}^{a}$, the lattice of analytically invariant subspaces of $B$ (see $[6, \S 2])$. Thus, we obtain the following

Corollary 5. An invertible bilateral weighted shift B in a Hilbert space of is always reflexive. Moreover, if $L$ is any bounded linear operator in of such that $\mathcal{J}_{L}$ $\supset \mathcal{J}_{B} \backslash \mathcal{L}_{B}^{a}$, then $L \in \mathfrak{U}_{B}$. 
Finally, we shall provide a counterexample for the following question:

Let $T \in \mathcal{L}(\mathcal{X})$ be a reflexive operator, $\mathcal{M} \in \mathcal{J}_{T}^{\prime}$, and $R=\left.T\right|_{\mathcal{M}}$; is $R$ a reflexive operator on $\mathcal{C} M$ ? $\left(\mathcal{J}_{T}^{\prime}=\right.$ lattice of $\mathfrak{A}_{T}^{\prime}$.)

In the case when $\mathcal{X}$ is finite dimensional, the answer is yes, as it follows from [1, Theorem 2]. This is no longer true if $\mathcal{X}$ is infinite dimensional; in fact, if $B$ is defined as in Theorem 4, with $a_{n}=1$, for $n \leq 0, a_{n}=2^{-n}$, for $n>0$, then $I^{+}=0, Q^{-}=1$ and $B$ is reflexive. Since $B$ is not invertible, $\mathfrak{A}_{B}^{\prime}=\mathfrak{A}_{B}$ (see [3]) and therefore $\mathcal{J}_{B}^{\prime}=\mathcal{Z}_{B}$. However $\left.B\right|_{\mathcal{A}_{0}}$ is a "Donoghue unilateral weighted shift" [2] and therefore, it is not reflexive (see [1]).

Similarly, it is possible to show that if $T$ is reflexive, $O M \in \mathcal{J}_{T}^{\prime}$ and $\bar{T}$ is the operator induced by $T$ on $\chi / \delta M$, then $\bar{T}$ is not necessarily reflexive.

\section{REFERENCES}

1. J. A. Deddens, Reflexive operators, Indiana Univ. Math. J. 20 (1971), 887-889.

2. R. Gellar, Cyclic vectors and parts of the spectrum of a weighted shift, Trans. Amer. Math. Soc. 146 (1969), 69-85. MR 41 \#4277b.

3. - Two sublattices of weighted shift invariant subspaces (to appear).

4. D. A. Herrero, Eigenvectors and cyclic vectors for bilateral weighted shifts, Rev. Un. Mat. Argentina (to appear).

5.—, Algebras de operadores que contienen una subálgebra de multiplicidad estricta finita, Rev. Un. Mat. Argentina (to appear).

6. D. A. Herrero and N. Salinas, Analytically invariant and bi-invariant subspaces (to appear).

7. Alan Lambert, Strictly cyclic operator algebras, Dissertation, University of Michigan, Ann Arbor, Mich., 1970.

8. - Strictly cyclic weighted shifts, Proc. Amer. Math. Soc. 29 (1971), 331-336. MR 43 \#970.

9. - Strictly cyclic operator algebras, Pacific J. Math. 39 (1971), 717-726.

10. C. E. Rickart, General theory of Banach algebras, University Series in Higher Math., Van Nostrand, Princeton, N.J., 1960. MR 22 \#5903.

11. D. Sarason, Invariant subspaces and unstarred operator algebras, Pacific J. Math. 17 (1966), 511-517. MR 33 \#590.

12. A. L. Shields and L. J. Wallen, The commutant of certain Hilbert space operators, Indiana Univ. Math. J. 20 (1971), 777-788.

Department of Mathematics, State University of New York at Albany, Albany, New YORK 12203

Department of Mathematics, Untversity of Kentucky, LeXington, Kentucky 40506 (Current address of Alan Lambert)

Current address (Domingo A. Herrero): Universidade Estadual de Campinas, Campinas, São Paulo, Brasil 